\title{
FLOW AND REACTIVE TRANSPORT IN POROUS MEDIA INDUCED BY WELL INJECTION: SIMILARITY SOLUTION
}

\author{
C.J. VAN DUIJN* AND PETER KNABNER**
}

\section{Introduction.}

Many situations arise in which hazardous chemicals, introduced into the subsurface, affect the quality of our drinking water supplies. Therefore it is of importance to understand and estimate the movement of such chemicals dissolved in the groundwater.

In this paper we consider the problem where chemicals are being injected into the soil by localized sources. Such a source can be a long, thin pipe buried into the soil, leading for homogeneous soils to a description in terms of two space dimensions, or some small localized source in the three dimensional space. By considering the appropriate half-space problem, also situations describing localized surface injection can be considered, see Figure 1. However by reflecting, we shall always select the domain

$$
\Omega_{\varepsilon}:=\left\{x \in \mathbb{R}^{N}:|x|>\varepsilon\right\},
$$

for $N=2$ and $N=3$ to model the porous medium, e.g. the soil or aquifer. Here $x[m]$ is a point in space and $\partial \Omega_{\varepsilon}=\left\{x \in \mathbb{R}^{N}:|x|=\varepsilon\right\}$ models the surface of the well, i.e. the source with radius $\varepsilon>0$.

Figure 1. Injection of contaminant into the soil $(N=2)$.

\footnotetext{
*Delft University of Technology, Department of Mathematics, P.O. Box 5031, NL 2600 GA Delft, NETHERLANDS.

**Institute for Applied Analysis and Stochastics, Hausvogteiplatz 5-7, D-O-1086 Berlin, GERMANY.
} 
The water flow regime is characterized by the water flux vector $\mathbf{q}[\mathrm{m} / \mathrm{s}]$ and the water content $\Theta\left[\mathrm{m}^{3} / \mathrm{m}^{3}\right]$ satisfying the conservation equation

$$
\partial_{t} \Theta=-\nabla \cdot \mathbf{q} \quad \text { for } \quad x \in \Omega_{\varepsilon}, t>0,
$$

where $t[s]$ denotes time.

The chemicals under discussion, such as organic herbicides or heavy metals, undergo various reactions. Adsorption to the solid soil particles as a retention/release reaction often is the most important factor with respect to the mobility of the chemical. Taking adsorption into account, the mass balance equation reads

$$
\partial_{t}(\Theta C+\rho S)+\nabla \cdot(C \mathbf{q}-\Theta D \nabla C)=0 \quad \text { for } \quad x \in \Omega_{\varepsilon}, t>0 .
$$

Here $C\left[\mathrm{~mol} / \mathrm{m}^{3}\right]$ denotes the concentration of dissolved chemical (per unit volume of fluid), $S[\mathrm{~mol} / \mathrm{kg}]$ the concentration of adsorbed chemical (per unit mass of porous skeleton), $\rho\left[\mathrm{kg} / \mathrm{m}^{3}\right]$ is the bulk density of the soil and $D\left[\mathrm{~m}^{2} / \mathrm{s}\right]$ a matrix, the sum of the molecular diffusion and mechanical dispersion (see e.g. Bear $[\mathrm{B}]$ ).

Often the adsorption reaction is fast compared to the water flow, such that a quasistationary approach is feasible, describing the reaction to be in equilibrium, i.e.

$$
S=\psi(C),
$$

where $\psi$, called the adsorption isotherm, can be derived from laboratory batch experiments. Typical examples of isotherms are (see e.g. Freeze and Cherry $[\mathrm{FC}]$ ):

$$
\begin{aligned}
& \psi(C)=k_{1} C^{p}, k_{1}>0,0<p<1 \text { (Freundlich) }, \\
& \psi(C)=\frac{k_{2} C}{1+k_{3} C}, k_{2}, k_{3}>0 \text { (Langmuir). }
\end{aligned}
$$

The example of the Freundlich isotherm shows that (1.3) is not only a nonlinear diffusionconvection equation in general, but may even be degenerate, since $\psi$ need not be differentiable at $C=0$. We will include these cases in our subsequent analysis.

Due to the injection of water at $\partial \Omega_{\varepsilon}$ the normal mass flux is given as a convective flux with the concentration $C_{e}$ of the chemical dissolved in the injected water, i.e.

$$
(C \mathbf{q}-\Theta D \nabla c) \cdot \boldsymbol{\nu}=C_{e} \mathbf{q} \cdot \boldsymbol{\nu} \quad \text { for } \quad x \in \partial \Omega_{\varepsilon}, t>0
$$

where $\boldsymbol{\nu}$ is the unit outward normal of $\Omega_{\varepsilon}$. Here $\boldsymbol{\nu}=-\mathbf{e}_{\mathbf{x}}$, with $\mathbf{e}_{\mathbf{x}}:=x /|x|$ being the unit vector in radial direction.

Equations (1.3), (1.4), (1.6) have to supplemented with an initial condition

$$
C(\cdot, 0)=C_{0} \quad \text { in } \Omega_{\varepsilon}
$$


Throughout this paper we shall consider $C_{e}$ and $C_{0}$ to be constant. The description of a contamination event leads to the property

$$
C_{e}>C_{0}
$$

One could also think of the reverse case

$$
C_{0}>C_{e}
$$

corresponding e.g. to a remediation event by flushing with clean water. As the analysis of both cases is substantially different, we will restrict here to (1.8) and postpone the investigation of (1.9).

Our concern is a detailed analysis of the concentration profiles in the vicinity of the well. To this end we assume some further simplifications. We consider the porous medium as homogeneous and either saturated or the water flow to be stationary. This leads to

$$
\rho>0, \Theta>0 \text { are constants }
$$

and thus from (1.2)

$$
\nabla \cdot \mathbf{q}=0 \text { for } x \in \Omega_{\varepsilon}, t>0
$$

If we assume the normal water flux at the well surface $\partial \Omega_{\varepsilon}$ to be uniform with a prescribed total rate $Q\left[m^{N} / s\right]$, possibly depending on time, then

$$
\mathbf{q}(x, t)=\frac{Q(t)}{\omega_{N} r^{N-1}} \mathbf{e}_{\mathbf{x}}
$$

where $r=|x|$ is the radial coordinate and $\omega_{N}$ the surface area of the unit ball in $\mathbb{R}^{N}$, i.e. $\omega_{N}=2(N-1) \pi$.

We are in particular interested in the dynamics near the well for small well radius $\varepsilon$. The conventional dispersion theories, relating $D$ to $\mathbf{q}$, would lead to unbounded dispersion coefficients for $\varepsilon \rightarrow 0$ (cf. Bear [B]). On the other hand we consider a homogeneous medium and thus expect that the dispersion and molecular diffusion coefficients are of the same order of magnitude. We describe this situation by assuming

$$
D>0 \text { is scalar and constant . }
$$

We non-dimensionalize the dependent variables in the following way: Set

$$
\begin{aligned}
u & :=\frac{C-C_{0}}{\delta C}, \quad \delta C:=C_{e}-C_{0}, \\
\beta(u): & =u+\frac{\rho}{\Theta \delta C}\left(\psi\left(\delta C u+C_{0}\right)-\psi\left(C_{0}\right)\right) .
\end{aligned}
$$


Then $u=u(x, t)$ satisfies

$$
\left\{\begin{array}{l}
\partial_{t} \beta(u)+\nabla \cdot\left(u \frac{\Lambda(t)}{|x|^{N-1}} \mathbf{e}_{\mathbf{x}}-D \nabla u\right)=0 \quad \text { for } \quad x \in \Omega_{\varepsilon}, t>0 \\
-D \nabla u \cdot \boldsymbol{\nu}=\frac{\Lambda(t)}{\varepsilon^{N-1}}(u-1) \quad \text { for } \quad x \in \partial \Omega_{\varepsilon}, t>0 \\
u(x, 0)=0 \text { for } \quad x \in \Omega_{\varepsilon}
\end{array}\right.
$$

Here

$$
\Lambda(t):=\frac{Q(t)}{\omega_{N} \Theta}
$$

Due to the radial symmetric flow field and the boundary conditions, we expect $u$ also to be radial symmetric and to satisfy

$$
\left(\tilde{P}_{\varepsilon}\right)\left\{\begin{array}{l}
\partial_{t} \beta(u)+\frac{1}{r^{N-1}} \partial_{r}\left(\Lambda(t) u-D r^{N-1} \partial_{r} u\right)=0 \text { for } r>\varepsilon, t>0 \\
D \partial_{r} u(\varepsilon, t)=\frac{\Lambda(t)}{\varepsilon^{N-1}}(u(\varepsilon, t)-1) \text { for } t>0 \\
u(r, 0)=0 \text { for } r>\varepsilon
\end{array}\right.
$$

To emphasize the role of $\varepsilon$ we denote its solutions by $u_{\varepsilon}=u_{\varepsilon}(r, t)$. With respect to $\beta$ we assume

$$
\begin{aligned}
& H_{\beta} 1: \beta \in C^{\infty}(0, \infty) \cap C([0, \infty)) \\
& H_{\beta} 2: \beta(0)=0, \beta^{\prime}(u)>0 \text { and } \beta^{\prime \prime}(u) \leq 0 \text { for } u>0 .
\end{aligned}
$$

These properties are in particular implied when using examples (1.5) for $\psi$.

To study Problem $\left(\tilde{P}_{\varepsilon}\right)$ for small well radius $\varepsilon$, it is reasonable to consider the limit $\varepsilon \rightarrow 0$. We conjecture the convergence, in a sense to be specified, of the solutions $u_{\varepsilon}$ to the solution $u=u(r, t)$ of

$$
\left(\tilde{P}_{0}\right)\left\{\begin{array}{l}
\partial_{t} \beta(u)+\frac{1}{r^{N-1}} \partial_{r}\left(\Lambda(t) u-D r^{N-1} \partial_{r} u\right)=0 \quad \text { for } \quad r>0, t>0 \\
u(0, t)=1 \quad \text { for } \quad t>0 \\
u(r, 0)=0 \quad \text { for } \quad r>0
\end{array}\right.
$$

This will be made rigorous, including rates of convergence, in a subsequent paper for two special cases of injection rates $Q(t)$, namely those for which the solution of $\left(\tilde{P}_{0}\right)$ exists in the form of a self-similar solution, i.e. only depending on the variable $\eta:=r t^{\alpha}$ with

$$
u(r, t)=f(\eta)
$$

Inserting $f$ into the differential equation and comparing terms, we see that for $\alpha$ the only possible choice is $\alpha=-1 / 2$ and for the injection rate

$$
Q(t)=Q_{1} t^{(N / 2)-1}
$$


for some $Q_{1}>0$.

The function $f=f(\eta), \eta=r / t^{1 / 2}$ then satisfies the boundary value problem

$$
(S D)\left\{\begin{array}{l}
\frac{1}{2} \eta^{N}\{\beta(f)\}^{\prime}+\left(D \eta^{N-1} f^{\prime}-a f\right)^{\prime}=0 \quad \text { for } 0<\eta<\infty \\
f(0)=1 \text { and } f(\infty)=0
\end{array}\right.
$$

where $^{\prime}=\partial_{\eta}$ and

$$
a:=\frac{Q_{1}}{\omega_{N} \Theta} .
$$

To study the existence of solutions of $(S D)$ and their properties is the purpose of this paper. In particular to investigate the limiting behaviour of the solution profiles for $D \rightarrow 0$ we use the form $(S D)$ and also for the outline of a numerical algorithm for the approximation of $f$. In those cases, where we consider a fixed dispersion coefficient $D$, we prefer a fully nondimensional form for the boundary value problem. We use $t D\left[\mathrm{~m}^{2}\right]$ instead of $t$. Then in (1.15), $D$ is substituted by 1 and $\Lambda(t)$ by $\lambda t^{N / 2-1}$, where $\lambda$ is a non-dimensional parameter given by

$$
\lambda:=\frac{Q_{1}}{\omega_{N} \Theta D^{N / 2}} .
$$

Problems $\left(\tilde{P}_{\varepsilon}\right)$ and $\left(\tilde{P}_{0}\right)$ and then substituted by

$$
\left(P_{\varepsilon}\right)\left\{\begin{array}{l}
\partial_{t} \beta\left(u_{\varepsilon}\right)+\frac{1}{r^{N-1}} \partial_{r}\left(\lambda t^{(N / 2)-1} u_{\varepsilon}-r^{N-1} \partial_{r} u_{\varepsilon}\right)=0 \text { for } r>\varepsilon, t>0, \\
\partial_{r} u_{\varepsilon}(\varepsilon, t)=\frac{\lambda}{\varepsilon^{N-1}} t^{(N / 2)-1}\left(u_{\varepsilon}(\varepsilon, t)-1\right) \quad \text { for } t>0, \\
u_{\varepsilon}(r, 0)=0 \text { for } r>0
\end{array}\right.
$$

and by $\left(P_{0}\right)$, consisting of the first and third equation and the boundary condition $u(0, t)=$ 1 for $t>0$. Analogously the equation for the self-similar solution now reads

$$
(S)\left\{\begin{array}{l}
\frac{1}{2} \eta^{N}\{\beta(f)\}^{\prime}+\left(\eta^{N-1} f^{\prime}-\lambda f\right)^{\prime}=0 \quad \text { for } 0<\eta<\infty, \\
f(0)=1 \text { and } f(\infty)=0 .
\end{array}\right.
$$

The outline of the sections is as follows: In Section 2 the unique existence of a solution of Problem $(S)$ and characteristic properties are investigated for $N=2$. The existence proof uses a shooting argument (for a transformed equation), which is also the basis of the numerical algorithm described in Section 5. For the behaviour near $\eta=0$ the value of the parameter $\lambda$ turns out to be crucial. The behaviour near $\eta=\infty$ is determined by the singular character of the reaction, leading to sharp fonts exactly in the case $1 / \beta \in L^{1}(0, \delta)$ for some $\delta>0$. Analogous results are sketched in Section 4 for $N=3$. Section 3 investigates the hyperbolic limit $D \rightarrow 0$ for the formulation of Problem $(S D)$. Finally Section 5 deals with an numerical algorithm for $(S D)$ and some examples. 


\section{The self-similar solution.}

In this section we study the existence, uniqueness and qualitative properties of solutions of the boundary value problem $(S)$. Except for some introductory observations, the cases $N=2$ and $N=3$ are treated separately. However only for $N=2$ the details of the proofs are given. The results for $N=3$ are summarized in Section 4 .

We start with the definition of a solution. For this we introduce the negative flux function

$$
F(\eta)=\eta^{N-1} f^{\prime}(\eta)-\lambda f(\eta), \quad \eta>0 .
$$

Definition 2.1. A function $f:[0, \infty) \rightarrow[0,1]$ is called a solution of Problem $(S)$ if

(i) $F, \beta(f)$ are absolutely continuous on $[0, \infty)$;

(ii)

$$
F^{\prime}+\frac{1}{2} \eta^{N}\{\beta(f)\}^{\prime}=0 \quad \text { a.e. on }(0, \infty)
$$

$$
f(0)=1 \quad \text { and } \quad f(\infty)=0 .
$$

From this definition we deduce

Proposition 2.2. Let $f$ be a solution of Problem $(S)$ and let $P=\{\eta>0: f(\eta)>0\}$. Then

(i) $f \in C^{\infty}(P)$;

(ii) $f^{\prime}<0$ on $P$;

(iii) $F(\eta) \rightarrow 0$ as $\eta \rightarrow \infty$.

Proof. (i) The continuity of $F$ and $f$ imply $f \in C^{1}((0, \infty))$ and $P$ open. Further, the boundary condition $f(0)=1$ gives that $P$ is non-empty. Thus each point $\eta_{0} \in P$ has a neighborhood $N \subset P$ such that $f$ is strictly positive on $N$. This implies $\beta(f) \in C^{1}(N)$ and, from equation (2.2), also $F \in C^{1}(N)$. Using this in (2.1) results in $f \in C^{2}(N)$. Continuing this process leads to the desired result.

(ii) This follows from a local uniqueness argument as in Atkinson \& Peletier [AP1, AP2].

(iii) Let $f(\eta)>0$ for all $\eta>0$. Then the monotonicity of $f$, together with (2.1) and (2.2), yields

$$
F(\eta)<0 \quad \text { and } \quad F^{\prime}(\eta)>0 \text { for all } \quad \eta>0 .
$$


Hence

$$
\lim _{\eta \rightarrow \infty} F(\eta)=F_{\infty} \leq 0 \quad \text { (exists) }
$$

We show that $F_{\infty}<0$ leads to a contradiction. Expression (2.1) gives

$$
\lim _{\eta \rightarrow \infty} \eta^{N-1} f^{\prime}(\eta) \rightarrow F_{\infty}
$$

For $N=2$, this contradicts directly the boundary condition $f(\infty)=0$. For $N=3$ we use (2.5) in equation (2.2) to find

$$
\lim _{\eta \rightarrow \infty} \frac{F^{\prime}(\eta)}{\eta}=-\frac{1}{2} \beta^{\prime}\left(0^{+}\right) F_{\infty}
$$

Now $F_{\infty}<0$ contradicts the asymptotic behaviour (2.4).

The monotonicity of $f$ means that $P$ consists of a single interval of the form

$$
P=(0, L) \quad \text { with } L \leq \infty \text {. }
$$

When $L=\infty$, we are dealing with a solution satisfying

$$
f(\eta)>0 \quad \text { and } \quad f^{\prime}(\eta)<0 \quad \text { for all } \quad \eta>0,
$$

and when $L<\infty$ (possibly), we have a solution of the form

$$
\begin{aligned}
& f(\eta)>0 \quad \text { and } f^{\prime}(\eta)<0 \text { for } 0<\eta<L, \\
& f(L)=f^{\prime}(L)=0 \\
& f(\eta)=0 \text { for } \eta>L .
\end{aligned}
$$

Whether $L<\infty$ or $L=\infty$ occurs, depends on the properties of the nonlinear term $\beta(f)$ near $f=0$. We will investigate this in the next section. Another point that requires attention concerns the behaviour of the solution near the origin, because there the equation has a singularity. We consider in detail the case $N=2$. 


\subsection{Structure of solution.}

Throughout this section we suppose that $f$ is a solution of Problem $(S)$ for $N=2$. With respect to the behaviour near $\eta=0$ we have

Proposition 2.3. $\lim _{\eta \downarrow 0} \eta^{1-\lambda} f^{\prime}(\eta)=-A$ (exists with $0<A<\infty$ ).

Proof. On the interval $(0, L)$ we may write the differential equation as

$$
\frac{f^{\prime \prime}}{f^{\prime}}=\frac{\lambda-1}{\eta}-\frac{1}{2} \eta \beta^{\prime}(f(\eta))
$$

Integrating this expression gives

$$
\eta_{2}^{1-\lambda} f^{\prime}\left(\eta_{2}\right)=\eta_{1}^{1-\lambda} f^{\prime}\left(\eta_{1}\right) e^{-\frac{1}{2} \int_{\eta_{1}}^{\eta_{2}} s \beta^{\prime}(f(s)) d s} \quad \text { for } \quad 0<\eta_{1}<\eta_{2}<L
$$

which implies the existence of the limit (fix $\eta_{2}>0$ and let $\eta_{1} \downarrow 0$ ).

Before we consider the behaviour of the solution near $\eta=L$, we first give the necessary and sufficient condition for which $L$ is finite; see also van Duijn \& Knabner [vDK1, vDK2] where similar questions for travelling waves were considered.

Proposition 2.4. $L<\infty \Leftrightarrow 1 / \beta \in L^{1}(0, \delta)$ for some $\delta>0$.

Proof. Suppose $1 / \beta \in L^{1}(0, \delta)$ and $L=\infty$. Integrating equation (2.2) from $\eta>0$ to $\infty$ gives

$$
\eta f^{\prime}(\eta)-\lambda f(\eta)=\frac{1}{2} \int_{\eta}^{\infty} s^{2}\{\beta(f(s))\}^{\prime} d s .
$$

Using the monotonicity of $\beta(f(\cdot))$ we estimate

$$
\eta f^{\prime}(\eta)-\lambda f(\eta)<-\frac{1}{2} \eta^{2} \beta(f(\eta))
$$

or

$$
-\frac{f^{\prime}(\eta)}{\beta(f(\eta))}>\frac{1}{2} \eta-\frac{f(\eta)}{\beta(f(\eta))} \frac{1}{\eta}
$$

for every $\eta>0$. Now fix $\eta_{0}>0$. Integrating (2.6) from $\eta_{0}$ to $\eta>\eta_{0}$ yields

$$
\int_{f(\eta)}^{f\left(\eta_{0}\right)} \frac{1}{\beta(s)} d s>\frac{1}{4}\left(\eta^{2}-\eta_{0}^{2}\right)-\int_{\eta_{0}}^{\eta} \frac{f(s)}{s \beta(f(s))} d s
$$


Since $\beta$ is concave we have

$$
\int_{f(\eta)}^{f\left(\eta_{0}\right)} \frac{1}{\beta(s)} d s>\frac{1}{4}\left(\eta^{2}-\eta_{0}^{2}\right)-\frac{f\left(\eta_{0}\right)}{\beta\left(f\left(\eta_{0}\right)\right)} \ln \left(\eta / \eta_{0}\right) .
$$

Letting $\eta \rightarrow \infty$, contradicts the integrability of $1 / \beta$. Next suppose $L<\infty$ and $1 / \beta \notin$ $L^{1}(0, \delta)$. Now we integrate equation (2.2) from some $0<\eta<L$ to $L$ and obtain

$$
\eta f^{\prime}(\eta)-\lambda f(\eta)=\frac{1}{2} \int_{\eta}^{L} s^{2}\{\beta(f(s))\}^{\prime} d s .
$$

In this expression we estimate for every $0<\eta<L$

$$
\eta f^{\prime}(\eta)-\lambda f(\eta)>-\frac{1}{2} L^{2} \beta(f(\eta))
$$

or

$$
-\frac{f^{\prime}(\eta)}{\beta(f(\eta))}<\frac{1}{2} \frac{L^{2}}{\eta}-\lambda \frac{f(\eta)}{\beta(f(\eta))} .
$$

Now fix $0<\eta_{0}<L$. Integrating (2.8) from $\eta_{0}$ to $\eta_{0}<\eta<L$ yields

$$
\int_{f(\eta)}^{f\left(\eta_{0}\right)} \frac{1}{\beta(s)} d s<\frac{1}{2} L^{2} \ln \left(\eta / \eta_{0}\right) .
$$

Letting $\eta \nearrow L$ in this expression, contradicts the non-integrability of $1 / \beta$.

To describe the behaviour of $f(\eta)$ near $\eta=L<\infty$ we introduce the function

$$
\Phi(f)=\int_{0}^{f} \frac{1}{\beta(s)} d s .
$$

Then we have

Proposition 2.5. $\lim _{\eta \uparrow L}\{\Phi(f(\eta))\}^{\prime}=-L / 2$.

Proof. From (2.7) we obtain for every $0<\eta<L$

$$
\eta f^{\prime}(\eta)-\lambda f(\eta)=-\frac{1}{2} \eta^{2} \beta(f(\eta))-\int_{\eta}^{L} s \beta(f(s)) d s
$$


or

$$
\{\Phi(f(\eta))\}^{\prime}+\frac{1}{2} \eta=\lambda \frac{f(\eta)}{\eta \beta(f(\eta))}-\frac{1}{\eta \beta(f(\eta))} \int_{\eta}^{L} s \beta(f(s)) d s .
$$

This equality implies

$$
\lim _{\eta \uparrow L}\{\Phi(f(\eta))\}^{\prime}=-\frac{1}{2} L+\frac{\lambda}{L \beta^{\prime}\left(0^{+}\right)}
$$

The concavity of $\beta$ gives $\beta^{\prime}\left(0^{+}\right) \leq \infty$. But $\beta^{\prime}\left(0^{+}\right)<\infty$ implies $1 / \beta \notin L^{1}(0, \delta)$. Hence $\beta^{\prime}\left(0^{+}\right)=\infty$, which yields the result.

Example 2.6. When considering a Freundlich isotherm we have, see also (1.5),

$$
\beta(f)=f+K f^{p} \quad \text { with } \quad K>0 \quad \text { and } \quad 0<p<1 .
$$

Clearly $1 / \beta \in L^{1}(0, \delta)$. For the behaviour of the solution near $L$ we find from

$$
\{\Phi(f(\eta))\}^{\prime}=\frac{f^{\prime}(\eta)}{f(\eta)+K f^{p}(\eta)}=\frac{1}{1-p} \frac{\left(f^{1-p}\right)^{\prime}(\eta)}{f^{1-p}(\eta)+K} \quad \text { for } 0<\eta<L
$$

that

$$
\lim _{\eta \uparrow L}\left(f^{1-p}\right)^{\prime}(\eta)=-\frac{1}{2}(1-p) K L
$$

In case of a Langmuir isotherm we have

$$
\beta(f)=f+\frac{K_{1} f}{1+K_{2} f}
$$

Hence $\beta^{\prime}\left(0^{+}\right)<\infty$ and consequently $L=\infty$.

\subsection{Existence, uniqueness and monotonicity.}

In view of the behaviour of the solution near $\eta=0$ we introduce here the transformation

$$
s=\frac{1}{\lambda} \eta^{\lambda} \quad \text { and } \quad g(s)=f(\eta)
$$

Then by Proposition 2.3, $g$ is differentiable up to $s=0$. Using this we study for $g$ the initial value problem

$$
(I V P)\left\{\begin{array}{l}
g^{\prime \prime}+C(\lambda) s^{\frac{2}{\lambda}-1}\{\beta(g)\}^{\prime}=0 \quad s>0, \\
g(0)=1, g^{\prime}(0)=-A,
\end{array}\right.
$$

where $C(\lambda)=\frac{1}{2} \lambda^{\frac{2}{\lambda}-1}$. For a given $A>0$, we denote the solution by $g(s ; A)$. The object here is to show that there exists a unique $A^{*}>0$ such that either $g\left(A^{*} ; s\right)>0$ 
for all $s \geq 0$ with $g\left(A^{*} ; \infty\right)=0$ (if $L=\infty$ ), or $g\left(A^{*} ; s\right)>0$ for $0 \leq s<L^{*}$ with $g\left(A^{*} ; L^{*}\right)=g^{\prime}\left(A^{*}, L^{*}\right)=0$ (if $\left.L<\infty\right)$. Here $L^{*}=\frac{1}{\lambda} L^{\lambda}$. Extending $g$ by zero in the latter case, it is then easily verified that the function

$$
f(\eta):=g\left(A^{*} ; \frac{1}{\lambda} \eta^{\lambda}\right), \eta \geq 0
$$

defines a solution in the sense of Definition 2.1.

To prove local existence for Problem (IVP) we first integrate (2.9) to obtain the integral representation

$$
g(s)=1-A \int_{0}^{s} \exp \left\{-C(\lambda) \int_{0}^{z} t^{\frac{2}{\lambda}-1} \beta^{\prime}(g(t)) d t\right\} d z=: \operatorname{Tg}(s)
$$

Then for $\delta>0$, sufficiently small (depending on $A$ ), the operator $T$ maps the set $X=$ $\left\{u \in C([0, \delta]): \frac{1}{2} \leq u \leq 1\right\}$ into itself and is a contraction. Consequently for any $A>0$, equation $(2.10)$ has a unique solution $g(A ; \cdot) \in X$. Then one shows that in fact $g(A ; \cdot) \in$ $C^{1}([0, \delta]) \cap C^{\infty}((0, \delta))$ and that on $(0, \delta), g$ satisfies equation $(2.9)$ with $g^{\prime}(A ; \cdot)<0$ and $g^{\prime \prime}(A ; \cdot)>0$. We can continue this solution for larger values of $s$ as long as $g$ remains positive. If the solution can be continued for all $s>0$, then the monotonicity implies the existence of

$$
\lim _{s \rightarrow \infty} g(A ; s)=: g(A ; \infty) \in(0,1)
$$

On the other hand, for $A$ large, the solution may exist only on a finite interval $\left(0, L_{A}\right)$ where

$$
g\left(A ; L_{A}\right)=0 \quad \text { and } \quad g^{\prime}\left(A ; L_{A}\right)<0 .
$$

Let

$$
S^{+}=\{A>0:(2.11) \text { holds }\}
$$

and

$$
S^{-}=\{A>0:(2.12) \text { holds }\} \text {. }
$$

Then

LEMMA 2.7. The sets $S^{+}$and $S^{-}$are non-empty, open and connected such that $\inf S^{+}=0$ and $\sup S^{-}=\infty$.

Proof. We first show that solutions $g(A ; \cdot)$ of Problem $(I V P)$ vary monotonically with the shooting parameter $A$. Let $0<A_{1}<A_{2}<\infty$ and let $g_{i}=g\left(A_{i} ; \cdot\right)$ for $i=1,2$. Clearly $g_{1}>g_{2}$ in a right neighborhood of $s=0$. Now suppose there exists $\hat{s}>0$ such that $g_{1}>g_{2}$ 
on $(0, \hat{s})$ and $g_{1}(\hat{s})=g_{2}(\hat{s})$. To reach a contradiction, we subtract the equations for $g_{1}$ and $g_{2}$, multiply the result by $s$ and integrate form $s=0$ to $s=\hat{s}$. Then

$$
\hat{s}\left(g_{1}-g_{2}\right)^{\prime}(\hat{s})-\frac{2}{\lambda} C(\lambda) \int_{0}^{\hat{s}} s^{\frac{2}{\lambda}-1}\left\{\beta\left(g_{1}\right)-\beta\left(g_{2}\right)\right\} d s .
$$

This is not possible because both terms have opposite sign. Hence if $A_{*} \in S^{+}$, then any $A<A_{*}$ belongs to $S^{+}$and clearly inf $S^{+}=0$. We construct a strictly positive lower bound on $[0, \infty)$ to show that $S^{+}$is non-empty. This bound follows from $(2.10)$ in which we use the observation that $\beta^{\prime}(g(t))>\beta^{\prime}(1)$ for $t>0$. Then

$$
g(s)>1-A \int_{0}^{s} \exp \left\{-\frac{\lambda}{2} C(\lambda) \beta^{\prime}(1) z^{\frac{2}{\lambda}}\right\} d z
$$

from which we deduce that

$$
\left(0,2^{1-\lambda}\left\{\beta^{\prime}(1)\right\}^{\frac{\lambda}{2}} / \Gamma\left(\frac{\lambda}{2}\right)\right) \subset S^{+} .
$$

where $\Gamma$ denotes the gamma function.

Next let $A^{*} \in S^{-}$. Because solutions do not intersect, we have for any $A>A^{*}$ that the corresponding solution becomes zero at $s=L_{A}<L_{A^{*}}$. If

$$
g^{\prime}\left(A ; L_{A}\right)<0
$$

then also $A \in S^{-}$. Now if $g^{\prime}\left(A ; L_{A}\right)=0$, then extending $g$ by zero would lead to a solution in the sense of Definition 2.1. Then we can apply the monotonicity argument from above to obtain a contradiction. Thus for $A>A^{*},(2.13)$ is the only possibility.

To show that $S^{-}$is non-empty we construct on upper bound which intersects the $s$-axis for $A$ sufficiently large. We obtain this bound by introducing the scaling

$$
t=A s \quad \text { and } \quad h(A ; t)=g(A ; s)
$$

and by considering for $h$ the resulting problem

$$
\left\{\begin{array}{l}
h^{\prime \prime}+C(\lambda) A^{-\frac{2}{\lambda}} t^{\frac{2}{\lambda}-1}\{\beta(h)\}^{\prime}=0 \quad t>0, \\
h(0)=1, h^{\prime}(0)=-1 .
\end{array}\right.
$$

Clearly $h^{\prime}<0$ and $h^{\prime \prime}>0$ on the interval of existence and

$$
h(A ; t) \geq \max \{(1-t), 0\}
$$


for all $t \geq 0$ and for all $A>0$. Integrating (2.14) twice gives

$$
h(t)=1-t-C(\lambda) A^{-\frac{2}{\lambda}} \int_{0}^{t}(t-z) z^{\frac{2}{\lambda}-1}\{\beta(h(z))\}^{\prime} d z .
$$

For $t \geq \frac{1}{2}$ we estimate the integrand in this expression with (2.15) to obtain

$$
(t-z) z^{\frac{2}{\lambda}-1}\{\beta(h(z))\}^{\prime} \geq\left\{\begin{array}{ll}
-(t-z) z^{\frac{2}{\lambda}-1} \beta^{\prime}\left(\frac{1}{2}\right) & \text { for } 0<z \leq \frac{1}{2} \\
t^{\frac{2}{\lambda}}\{\beta(h(z))\}^{\prime}(\lambda \leq 2) \\
t\left(\frac{1}{2}\right)^{\frac{2}{\lambda}-1}\{\beta(h(z))\}^{\prime} \quad(\lambda>2)
\end{array}\right\} \quad \text { for } \frac{1}{2} \leq z \leq t
$$

This leads to

$$
h(z) \leq 1-t+\frac{\lambda}{2} C(\lambda) A^{-\frac{2}{\lambda}} \beta^{\prime}\left(\frac{1}{2}\right)\left(\frac{1}{2}\right)^{\frac{2}{\lambda}} t+C(\lambda) A^{-\frac{2}{\lambda}} \beta(1) d(\lambda ; t) t,
$$

where

$$
d(\lambda ; t)= \begin{cases}t^{\frac{2}{\lambda}-1} & (\lambda \leq 2), \\ \left(\frac{1}{2}\right)^{\frac{2}{\lambda}-1} & (\lambda>2) .\end{cases}
$$

The right-hand side in (2.16) becomes negative for the appropriate choice of $A$ (large) and $t>1$. Hence for all sufficiently large $A$, the solutions $g(A ; s)$ vanish at $s=L_{A}$ where $g^{\prime}\left(A ; L_{A}\right) \leq 0$. Using again the monotonicity argument one finds that there cannot exist two of such solutions which both have a zero derivative when they vanish. This proves that $S^{-}$is nonempty. The upperbound (2.10) also implies that $\sup S^{-}=\infty$.

Finally, the continuous dependence for Problem $(I V P)$ gives that both $S^{+}$and $S^{-}$are open.

The properties of the sets $S^{+}$and $S^{-}$imply that there exists $A^{*} \in(0, \infty)$ such that

$$
\sup S^{+} \leq A^{*} \leq \inf S^{-}
$$

Considering the solution $g\left(A^{*} ; s\right)$ we have either

$$
(I)\left\{\begin{array}{l}
g\left(A^{*} ; \cdot\right)>0, g^{\prime}\left(A^{*} ; \cdot\right)<0 \\
g^{\prime \prime}\left(A^{*} ; \cdot\right)>0 \text { on }(0, \infty)
\end{array}\right.
$$

or there exists $L^{*}>0$ such that

$$
(I I)\left\{\begin{array}{l}
g\left(A^{*} ; \cdot\right)>0 \text { on }\left(0, L^{*}\right), \\
g\left(A^{*} ; L^{*}\right)=g^{\prime}\left(A^{*}, L^{*}\right)=0 .
\end{array}\right.
$$

Applying once more the monotonicity argument, it follows that the value of $A^{*}$ is uniquely determined. Extending $g$ by zero in case (II) we obtain 
ThEOREM 2.8. Problem $(S)$ has a unique solution $f$, given by $f(\eta)=g\left(A^{*} ; \frac{1}{\lambda} \eta^{\lambda}\right)$ for $\eta \geq 0$.

Problem $(S)$ can be solved explicitly when $\lambda=2$. We work out the details in the next example.

Example 2.9. Taking $\lambda=2$, reduces equation (2.9) to

$$
g^{\prime \prime}+\frac{1}{2}\{\beta(g)\}^{\prime}=0 \quad \text { for } \quad s>0 .
$$

Integration gives

$$
g^{\prime}+\frac{1}{2} \beta(g)=-A+\frac{1}{2} \beta(1)
$$

Choosing $A=\frac{1}{2} \beta(1)$ and using $\beta(0)=0$, implies that $g^{\prime}$ vanishes whenever $g$ vanishes. Hence we find the expressions

$$
\int_{g(s)}^{1} \frac{1}{\beta(t)} d t=\frac{1}{2} s \quad \text { and } \quad \int_{f(\eta)}^{1} \frac{1}{\beta(t)} d t=\frac{1}{4} \eta^{2}
$$

When the function $\beta$ is differentiable upto $s=0$, i.e. $\beta^{\prime}\left(0^{+}\right)<\infty$, we have precise information about the asymptotic behaviour of $f(\eta)$ as $\eta \rightarrow \infty$.

THEOREM 2.10. Let $\beta$ satisfy $H_{\beta 1-2}$ such that $\beta^{\prime} \in C^{\alpha}([0,1])$, with $\alpha \in(0,1)$. Then given any $\eta_{0}>0$, there exists constants $K_{1}, K_{2}$ such that

$$
K_{1} \leq f(\eta) /\left(\int_{\frac{1}{\lambda} \eta^{\lambda}}^{\infty} e^{-\frac{1}{2} C(\lambda) \beta^{\prime}(0) t^{\frac{2}{\lambda}}} d t\right) \leq K_{2}
$$

for all $\eta \geq \eta_{0}$.

Proof. We construct the estimates for the transformed function $g$. Equation (2.9) implies

$$
-C(\lambda) s^{\frac{2}{\lambda}-1} \beta^{\prime}(0) \leq \frac{g^{\prime \prime}}{g^{\prime}}=-C(\lambda) s^{\frac{2}{\lambda}-1} \beta^{\prime}(g) \leq-C(\lambda) s^{\frac{2}{\lambda}-1} \beta^{\prime}(1) .
$$

Integrating given for $s \geq s_{0}>0$

$$
-\frac{\lambda}{2} C(\lambda) \beta^{\prime}(0)\left(s^{\frac{2}{\lambda}}-s_{0}^{\frac{2}{\lambda}}\right) \leq \ln \left(\frac{g^{\prime}(s)}{g^{\prime}\left(s_{0}\right)}\right) \leq-\frac{\lambda}{2} C(\lambda) \beta^{\prime}(1)\left(s^{\frac{2}{\lambda}}-s_{0}^{\frac{2}{\lambda}}\right)
$$


and once more

$$
K_{1} \int_{s}^{\infty} e^{-\frac{\lambda}{2} C(\lambda) \beta^{\prime}(0) t^{\frac{2}{\lambda}}} d t \leq g(s) \leq K_{2}^{*} \int_{s}^{\infty} e^{-\frac{\lambda}{2} C(\lambda) \beta^{\prime}(1) t^{\frac{2}{\lambda}}} d t
$$

Next we improve the upperbound by writing

$$
\frac{g^{\prime \prime}}{g^{\prime}}=-C(\lambda) \beta^{\prime}(0) s^{\frac{2}{\lambda}-1}+C(\lambda) s^{\frac{2}{\lambda}-1}\left\{\beta^{\prime}(0)-\beta^{\prime}(g)\right\}
$$

Using the upperbound from (2.18) and the Hölder continuity of the derivative $\beta^{\prime}$, it follows that the second term on the right in (2.19) is integrable on $\left[s_{0}, \infty\right)$. Hence

$$
\frac{g^{\prime \prime}}{g^{\prime}}<-C(\lambda) \beta^{\prime}(0) s^{\frac{2}{\lambda}-1}+C^{*}, \quad s \geq s_{0},
$$

for some $C^{*}>0$. Integrating this expression twice gives the improved upperbound

$$
g(s) \leq K_{2} \int_{s}^{\infty} e^{-\frac{\lambda}{2} C(\lambda) \beta^{\prime}(0) t^{\frac{2}{\lambda}}} d t
$$

for some $K_{2}>0$. Returning to the variable $\eta$ proves the result.

For later purpose, in particular when studying the convergence of solutions of the time dependent problem in case of singular reactions, we regularize the function $\beta$. We put

$$
\beta_{n}(s)=\beta\left(s+\frac{1}{n}\right)-\beta\left(\frac{1}{n}\right) s \geq 0, n \in \mathbb{N}
$$

Then $\beta_{n}$ satisfies again $H_{\beta_{1,2}}$ and in addition $\beta_{n} \in C^{\infty}([0, \infty))$. It leads to the regularized problems

$$
\left(S_{n}\right)\left\{\begin{array}{l}
f^{\prime \prime}+\frac{1-\lambda}{\eta} f^{\prime}+\eta\left\{\beta_{n}(f)\right\}^{\prime}=0 \quad \eta>0, \\
f(0)=1, \quad f(\infty)=0
\end{array}\right.
$$

By Theorem (2.8) Problem $\left(S_{n}\right)$ has a unique solution $f_{n}$, which is positive, smooth and strictly decreasing on $(0, \infty)$. Furthermore, each $f_{n}$ satisfies the asymptotic behaviour from Theorem (2.10) with $\beta^{\prime}(0)$ replaced by $\beta_{n}^{\prime}(0)=\beta^{\prime}\left(\frac{1}{n}\right)$. We have further

THEOREM 2.11. Considering the approximations $(2.20)$, we have $f_{n}(\eta) \rightarrow f(\eta)$ as $n \rightarrow \infty$, uniformly in $\eta \geq 0$.

Proof. For each $n \in \mathbb{N}$, the transformed functions $g_{n}$ satisfy

$$
\left\{\begin{array}{l}
g^{\prime \prime}+C(\lambda) s^{\frac{2}{\lambda}-1}\left\{\beta_{n}(g)\right\}^{\prime}=0 \quad s>0 \\
g(0)=1, \quad g(\infty)=0
\end{array}\right.
$$


Setting $z_{n}=g_{n}+\frac{1}{n}$ and using (2.20) we find that each $z_{n}$ is a solution of

$$
\left\{\begin{array}{l}
z^{\prime \prime}+C(\lambda) s^{\frac{2}{\lambda}-1}\{\beta(z)\}^{\prime}=0 \quad s>0, \\
z(0)=1+\frac{1}{n}, \quad z(\infty)=\frac{1}{n} .
\end{array}\right.
$$

Applying again the monotonicity argument we find

$$
z_{n} \geq z_{n+1} \geq g \quad \text { on }[0, \infty), \quad \text { for all } n \in \mathbb{N}
$$

Hence

$$
\lim _{n \rightarrow \infty} z_{n} \searrow \underline{z} \geq g \quad \text { pointwise on }(0, \infty)
$$

and thus

$$
\lim _{n \rightarrow \infty} g_{n} \rightarrow \underline{z} \geq g \quad \text { pointwise on }(0, \infty) .
$$

Next multiply equation $(2.21)$ by $s$ and integrate the result. For any $s>0$ and $n \in \mathbb{N}$ there results

$$
s g_{n}^{\prime}(s)=g_{n}(s)-1-C(\lambda) s^{\frac{2}{\lambda}} \beta_{n}\left(g_{n}(s)\right)+\frac{2}{\lambda} C(\lambda) \int_{0}^{s} t^{\frac{2}{\lambda}-1} \beta_{n}\left(g_{n}(t)\right) d t .
$$

Thus for any given $K \Subset(0, \infty)$, there exists $M>0$ such that

$$
-M \leq g_{n}^{\prime}<0 \quad \text { on } K, \quad \text { for all } n \in \mathbb{N} .
$$

By equicontinuity,

$$
g_{n} \rightarrow \underline{z} \text { in } C(K),
$$

along some subsequence $n \nearrow \infty$. In fact, since $g \leq \underline{z} \leq 1$ on $[0, \infty)$, we obtain $\underline{z} \in$ $C([0, \infty))$ and $\underline{z}(0)=1$. The monotonicity of the sequence $\left\{z_{n}\right\}$ gives $\underline{z}(\infty)=0$ and

$$
g_{n} \rightarrow \underline{z} \text { in } C([0, \infty)),
$$

for the entire sequence $n \nearrow \infty$ (apply Dini's Theorem to $\left\{z_{n}\right\}$ and use the asymptotic behaviour (upperbound in (2.18)). Passing to the limit in expressing (2.23) gives $\underline{z} \in$ $C^{1}((0, \infty))$ and for all $s>0$

$$
s \underline{z}^{\prime}(s)=\underline{z}(s)-1-C(\lambda) s^{\frac{2}{\lambda}} \beta(\underline{z}(s))+\frac{2}{\lambda} C(\lambda) \int_{0}^{s} t^{\frac{2}{\lambda}-1} \beta(\underline{z}(t)) d t .
$$

Since $\underline{z}$ decays exponentially fast at infinity, we obtain from $(2.24)$ that $\lim _{s \rightarrow \infty} s \underline{z}^{\prime}(s)=l$ (exists). Clearly $l=0$ since $\underline{z}(\infty)=0$. Therefore

$$
\frac{2}{\lambda} C(\lambda) \int_{0}^{\infty} t^{\frac{2}{\lambda}-1} \beta(\underline{z}(t)) d t=1 \text {. }
$$

The same identity holds for $g$. Then we conclude from $\underline{z} \geq g$, that in fact $\underline{z}=g$ on $[0, \infty)$. This completes the proof of the theorem. 


\section{The hyperbolic limit.}

To study the limit $D \searrow 0$, we cannot use the scaling of the variables as was done to obtain Problem $(S)$. Instead we return to the non-dimensionless formulation leading to Problem $(S D)$, which we give here for $N=2$ :

$$
(S D)\left\{\begin{array}{l}
\left\{D \eta f^{\prime}-a f\right\}^{\prime}+\frac{1}{2} \eta^{2}\{\beta(f)\}^{\prime}=0 \quad \eta>0 \\
f(0)=1, \quad f(\infty)=0 .
\end{array}\right.
$$

From the theory developed in Section 2 we conclude that for each $D>0$, this problem has a unique solution $f_{D}$ which satisfies

(i) $0 \leq f_{D} \leq 1 \quad$ on $[0, \infty)$;

(ii) $\left\|f_{D}^{\prime}\right\|_{L^{1}((0, \infty))}=1$ ( by the monotonicity of $f_{D}$ );

(iii) $\int_{0}^{\infty} \eta \beta\left(f_{D}(\eta)\right) d \eta=a($ mass-conservation ).

We use $W^{1,1}(K) \Subset L^{p}(K)$ for $1 \leq p<\infty$ and for $K \Subset(0, \infty)$, to obtain that for a sequence $D \searrow 0$ there exists a function $\hat{f} \in L^{\infty}((0, \infty))$ such that

$$
0 \leq \hat{f} \leq 1 \quad \text { a.e. on }[0, \infty),
$$

and a subsequence, denoted again by $D \searrow 0$, along which

$$
f_{D} \rightarrow \hat{f} \text { in } L^{p}(K) \text { and } f_{D} \rightarrow \hat{f} \text { a.e. on }(0, \infty) .
$$

The monotonicity of the functions in the approximating sequence implies that $\hat{f}$, possibly redefined on a set of measure zero, is monotone on $(0, \infty)$. Further it follows from (3.3) and $\hat{f} \geq 0$ that

$$
\int_{0}^{\infty} \eta \beta(\hat{f}(\eta)) d \eta=a
$$

Hence $\hat{f}(\infty)=0$. To obtain an equation for the limit $\hat{f}$, we introduce the weak form of $(3.1)$, i.e.

$$
\int_{0}^{\infty}\left\{\left(D \eta f_{D}^{\prime}-a f_{D}\right) \varphi^{\prime}+\beta\left(f_{D}\right)\left(\eta \varphi+\frac{1}{2} \eta^{2} \varphi^{\prime}\right)\right\} d \eta=0
$$

with $\varphi \in C_{0}^{\infty}((0, \infty))$. Passing to the limit in this expression gives

$$
\int_{0}^{\infty}\left\{\eta \beta(\hat{f}) \varphi+\left(\frac{1}{2} \eta^{2} \beta(\hat{f})-a \hat{f}\right) \varphi^{\prime}\right\} d \eta=0
$$


for all $\varphi \in C_{0}^{\infty}((0, \infty))$. Hence

$$
\frac{1}{2} \eta^{2} \beta(\hat{f})-a \hat{f} \text { is absolutely continuous on }(0, \infty)
$$

and

$$
\frac{1}{2} \eta_{2}^{2} \beta\left(\hat{f}\left(\eta_{2}\right)\right)-a \hat{f}\left(\eta_{2}\right)-\frac{1}{2} \eta_{1}^{2} \beta\left(\hat{f}\left(\eta_{1}\right)\right)+a \hat{f}\left(\eta_{1}\right)=\int_{\eta_{1}}^{\eta_{2}} \eta \beta(\hat{f}(\eta)) d \eta
$$

for any $0<\eta_{1}<\eta_{2}<\infty$. Letting $\eta_{2} \rightarrow \infty$ in this expression shows that

$$
\lim _{\eta \rightarrow \infty} \eta^{2} \beta(\hat{f}(\eta))=\ell \in \mathbb{R}(\text { exists })
$$

Now suppose $\ell \neq 0$. Then

$$
\eta \beta(\hat{f}(\eta)) \approx \ell / \eta \text { as } \eta \rightarrow \infty
$$

contradicting the mass conservation equation (3.4). Hence $\ell=0$ and (3.5) gives, with $\eta_{2} \rightarrow \infty$

$$
-\frac{1}{2} \eta^{2} \beta(\hat{f}(\eta))+a \hat{f}(\eta)=\int_{\eta}^{\infty} s \beta(\hat{f}(s)) d s
$$

This shows that

$$
\lim _{\eta \downarrow 0} \hat{f}(\eta)=1
$$

Now suppose $\hat{f}$ has a jump discontinuity for some $\eta_{s}>0$, with

$$
\hat{f}^{+(-)}=\lim _{\eta \uparrow(\downarrow) \eta_{s}} \hat{f}(\eta)
$$

Then the continuity of the right-hand side in (3.6) gives the relation

$$
\eta_{s}^{2}=2 a \frac{\hat{f}^{+}-\hat{f}^{-}}{\beta\left(\hat{f}^{+}\right)-\beta\left(\hat{f}^{-}\right)}
$$

which is the well-known Rankine-Hugoniot shock condition, e.g. see Whitham [W]. The concavity of $\beta$, applied to condition (3.8), yields that there exists at most one value of $\eta$ where a discontinuity or shock can occur. By the monotonicity of $\hat{f}$ and (3.7) there are two 
possibilities. Either $\hat{f} \in C([0, \infty))$ or there exists $\eta_{s}>0$ such that $\hat{f} \in C\left(\left[0, \eta_{s}\right) \cup\left(\eta_{s}, \infty\right)\right)$ with $\hat{f}^{+}>\hat{f}^{-}$at $\eta_{s}$. Now suppose that for some interval $I \in(0, \infty)$ we have

$$
\hat{f}>0 \text { and strictly decreasing on } I, \quad \hat{f} \in C(I) .
$$

Then from (3.6)

$$
-\frac{1}{2} \eta^{2} \beta(\hat{f})+a \hat{f} \in C^{1}(I)
$$

and

$$
\left\{-\frac{1}{2} \eta^{2} \beta(\hat{f})+a \hat{f}\right\}^{\prime}=-\eta \beta(\hat{f}) \text { on } I .
$$

Using once more the continuity of $\hat{f}$, we obtain for each $\eta \in I$

$$
\lim _{\Delta \rightarrow 0} \frac{\hat{f}(\eta+\Delta)-\hat{f}(\eta)}{\Delta}\left\{a-\frac{1}{2} \eta^{2} \frac{\beta(\hat{f}(\eta+\Delta))-\beta(\hat{f}(\eta))}{\hat{f}(\eta+\Delta)-\hat{f}(\eta)}\right\}=0 .
$$

The term between brackets converges, for $\Delta \rightarrow 0$, to

$$
a-\frac{1}{2} \eta^{2} \beta^{\prime}(\hat{f}(\eta))
$$

Note that this is a strictly decreasing function of $\eta$ (since $\beta^{\prime \prime} \leq 0$ ), taking on the value $a$ at $\eta=0$ and converging to $-\infty$ for $\eta \rightarrow \infty$. Hence there is a unique $\tilde{\eta}>0$ such that $a-\frac{1}{2} \tilde{\eta}^{2} \beta^{\prime}(\hat{f}(\tilde{\eta}))=0$. Using this observation in (3.9) gives

$$
\hat{f} \text { differentiable with } \hat{f}^{\prime}=0 \text { on } I \backslash\{\tilde{\eta}\}
$$

and, since $\hat{f} \in C(I)$,

$$
\hat{f}=\text { constant on } I \text {, }
$$

contradicting the strict monotonicity of $\hat{f}$ on $I$. It leaves for $\hat{f}$ as only possibility

$$
\hat{f}(\eta)=\left\{\begin{array}{ll}
1 & 0 \leq \eta<\eta_{s} \\
0 & \eta_{s}<\eta<\infty
\end{array} \quad \text { with } \eta_{s}=\left\{\frac{2 a}{\beta(1)}\right\}^{1 / 2}\right.
$$

By the uniqueness of the limit function, the solutions $f_{D}$ corresponding to the entire sequence $D \searrow 0$ converge to $\hat{f}$. Hence we have shown

Theorem 3.1. Let $f_{D}$ be the solution of Problem $(S D)$ for $D>0$. Then along any sequence $D \searrow 0$,

$$
f_{D} \rightarrow \hat{f} \text { a.e. on }(0, \infty)
$$

with $\hat{f}$ given by (3.10).

Remark 3.2. In case of a singular reaction, leading to $f_{D}(\eta)>0$ for $0 \leq \eta<L_{D}$ and $f_{D}(\eta)=0$ for $\eta \geq L_{D}$, it follows from the mass-conservation equation (3.3) that $L_{D}>\eta_{s}$ for every $D>0$. 


\section{Results for $N=3$.}

We recall Problem $(S)$ for $N=3$

$$
(S)\left\{\begin{array}{l}
\left(\eta^{2} f^{\prime}-\lambda f\right)^{\prime}+\frac{1}{2} \eta^{3}\{\beta(f)\}^{\prime}=0, \quad \eta>0 \\
f(0)=1, \quad f(\infty)=0 .
\end{array}\right.
$$

Multiplying equation (4.1) by $\exp (\lambda / \eta)$ yields

$$
\left\{\eta^{2} e^{\frac{\lambda}{\eta}} f^{\prime}\right\}^{\prime}+\frac{1}{2} \eta^{3} e^{\frac{\lambda}{\eta}}\{\beta(f(\eta))\}^{\prime}=0
$$

from which we deduce for the behaviour near $\eta=0$

$$
\lim _{\eta \downarrow 0} \eta^{2} e^{\frac{\lambda}{\eta}} f^{\prime}(\eta)=-A(\text { exists with } 0<A<\infty) .
$$

Consequently

$$
\lim _{\eta \downarrow 0} f^{\prime}(\eta)=0 \quad \text { for all } \lambda>0
$$

As in the two dimensional setting we have here

$$
f^{\prime}(\eta)<0 \text { whenever } f(\eta)>0
$$

which implies again that the set

$$
P=\{\eta>0: f(\eta)>0\}
$$

is of the form

$$
P=(0, L) \text { with } L \leq \infty .
$$

The characterization for finite $L$ is as in Proposition 2.4, i.e.

$$
L<\infty \Leftrightarrow 1 / \beta \in L^{1}(0, \delta) \text { for some } \delta>0
$$

When $L<\infty$, the behaviour of $f(\eta)$ for $\eta$ near $L$ is given by Proposition 2.5.

To prove the existence result we introduce the transformation

$$
s=e^{-\frac{\lambda}{\eta}} \text { and } g(s)=f(\eta)
$$

and study the initial value problem

$$
(I V P)\left\{\begin{array}{l}
g^{\prime \prime}+\frac{1}{2} \lambda^{2}\left(\frac{-1}{\ln s}\right)^{3} \frac{1}{s}\{\beta(g)\}^{\prime}=0 \quad 0<s<1 \\
g(0)=1, g^{\prime}(0)=-A .
\end{array}\right.
$$


Again we look for decreasing and concave solutions of this problem. By the method of Section 2.2 we find that there exists a unique $A^{*}>0$ and a unique solution $g\left(A^{*} ; s\right)$ such that either

$$
I\left\{\begin{array}{l}
g\left(A^{*} ; \cdot\right)>0 \text { on }(0,1), \\
g\left(A^{*} ; 1\right)=g^{\prime}\left(A^{*} ; 1\right)=0,
\end{array}\right.
$$

or there exists $L^{*} \in(0,1)$ such that

$$
I I\left\{\begin{array}{l}
g\left(A^{*} ; \cdot\right)>0 \text { on }\left(0, L^{*}\right), \\
g\left(A^{*} ; L^{*}\right)=g^{\prime}\left(A^{*} ; L^{*}\right)=0 .
\end{array}\right.
$$

In the latter case we extend the solution by zero on $(0,1)$. As a result we find that

$$
f(\eta):=g\left(A^{*} ; e^{-\frac{\lambda}{\eta}}\right) \quad \eta \geq 0,
$$

is the unique solution of Problem $(S)$.

Example 4.1. Let the adsorption isotherm $\psi$ be linear. Then

$$
\beta(f)=f+K f \text { with } K>0,
$$

and for Problem (IVP) results

$$
\left\{\begin{array}{l}
g^{\prime \prime}+\frac{1}{2} \lambda^{2}(1+K)\left(\frac{-1}{\ln s}\right)^{3} \frac{1}{s} g^{\prime}=0 \quad 0<s<1, \\
g(0)=1, \quad g^{\prime}(0)=-A
\end{array}\right.
$$

Integrating the equation twice yields

$$
g(s)=1-A \int_{0}^{s} e^{-\frac{1}{4} \lambda^{2}(1+K)\left\{\frac{1}{\ln t}\right\}^{2}} d t .
$$

Taking

$$
A^{*}=1 / \int_{0}^{1} e^{-\frac{1}{4} \lambda^{2}(1+K)\left\{\frac{1}{\ln t}\right\}^{2}} d t,
$$

gives a positive, decreasing and concave solution on $(0,1)$ which satisfies

$$
g\left(A^{*} ; 1\right)=g^{\prime}\left(A^{*} ; 1\right)=0 .
$$

In terms of the original similarity variable the solution reads

$$
f(\eta)=1-A^{*} \int_{0}^{e^{-\lambda / \eta}} e^{-\frac{1}{4} \lambda^{2}(1+K)\left\{\frac{1}{\ln t}\right\}^{2}} d t .
$$


To study the hyperbolic limit $D \searrow 0$, we return to Problem $(S D)$ for $N=3$ :

$$
(S D)\left\{\begin{array}{l}
\left(D \eta^{2} f^{\prime}-a f\right)^{\prime}+\frac{1}{2} \eta^{3}\{\beta(f)\}^{\prime}=0 \quad \eta>0 \\
f(1)=1, \quad f(\infty)=0
\end{array}\right.
$$

Note that the dependent variable $\eta$ in Problems $(S D)$ and $(S)$ is different: $\eta=r / \sqrt{t}[\mathrm{~m} / \mathrm{s}]$ in Problem $(S D)$, whereas $\eta=r / \sqrt{D t}[-]$ in Problem $(S)$.

Any solution $f_{D}$ of Problem $(S D)$ satisfies the mass- conservation identity

$$
\int_{0}^{\infty} \eta^{2} \beta\left(f_{D}(\eta)\right) d \eta=\frac{2}{3} a
$$

Going through the procedure of Section 3, we obtain that for any sequence $D \searrow 0$

$$
f_{D} \rightarrow \hat{f} \text { a.e. on }(0, \infty)
$$

where

$$
\hat{f}(\eta)=\left\{\begin{array}{ll}
1 & 0 \leq \eta<\eta_{s} \\
0 & \eta_{s}<\eta<\infty
\end{array} \quad \text { with } \eta_{s}=\left\{\frac{2 a}{\beta(1)}\right\}^{1 / 2}\right.
$$




\section{Numerical Approximation.}

In this section we describe an algorithm to approximate the solutions of Problem $(S D)$ and indicate some examples. We only consider here $N=2$. Based on Section 4 an analogous algorithm for $N=3$ can be designed. The numerical procedure is strongly related to the existence proof from Section 2.2. It is shown there (by means of the transformation $\left.s=\frac{1}{\lambda} \eta^{\lambda}\right)$ that the situation is as follows.

The solution of Problem $(S D)$ is characterized by a value $A^{*}>0$ such that

$$
\lim _{\eta \downarrow 0} \eta^{1-\lambda} f^{\prime}(\eta)=-A^{*}
$$

and we have, setting

$$
S^{+}:=\left[0, A^{*}\right), \quad S^{-}:=\left(A^{*}, \infty\right),
$$

that for $A \in S^{+}$the solution of the initial value problem

$$
\left(I V P_{\eta}\right)\left\{\begin{array}{l}
\frac{1}{2} \eta^{N}\{\beta(f)\}^{\prime}+\left(-a f+D \eta^{N-1} f^{\prime}\right)^{\prime}=0 \\
f(0)=1, \quad \lim _{\eta \downarrow 0} \eta^{1-\lambda} f^{\prime}(\eta)=-A,
\end{array}\right.
$$

satisfies

$$
\lim _{\eta \rightarrow \infty} f(\eta)>0
$$

and for $A \in S^{-}$the solution of $\left(I V P_{\eta}\right)$ satisfies

$$
f\left(\eta_{A}\right)=0, f^{\prime}\left(\eta_{A}\right)<0 \quad \text { for some } \quad \eta_{A}>0 .
$$

This shows the convergence of the shooting algorithm (if performed exactly):

Shooting Algorithm

(1) Choose $A_{l}^{0} \in S^{-}, A_{u}^{0} \in S^{+}, j:=0$.

(2) $A:=\frac{1}{2}\left(A_{l}^{j}+A_{u}^{j}\right), j:=j+1$.

(3) Compute the solution of $\left(I V P_{\eta}\right)$.

(4) If $A \in S^{+}$,

$$
A_{u}^{j}:=A, \quad A_{l}^{j}:=A_{l}^{j-1}, \quad \text { goto (2). }
$$

(5) If $A \in S^{-}$,

$$
A_{l}^{j}:=A, \quad A_{u}^{j}:=A_{u}^{j-1}, \quad \text { goto }(2) .
$$

(6) Stop. 
This algorithm either finds the solution in finitely many steps or generates sequences $A_{l}^{j} \in S^{-}, A_{u}^{j} \in S^{+}$, such that

$$
\left|A_{l}^{j}-A_{u}^{j}\right|=2^{-j}\left|A_{l}^{0}-A_{u}^{0}\right| \rightarrow 0 \text { for } j \rightarrow \infty,
$$

and $A^{*} \in\left(A_{u}^{j}, A_{l}^{j}\right)$. Several steps of the algorithm cannot be performed exactly. To approximate the solution of $\left(I V P_{\eta}\right)$ we integrate the equation as in the proof of Proposition 2.3. This incorporates the shooting parameter $A$ :

$$
f^{\prime}(\eta)=-A \eta^{\frac{a}{D}-1} \exp \left(-\frac{1}{2 D} \int_{0}^{\eta} s \beta^{\prime}(f(s)) d s\right), f(0)=1 .
$$

We approximate the solution of (5.4) by the third order Adams-Bashforth method. The integrands are approximated by the trapezoidal rule. The procedure is started by extrapolation steps starting from $\eta=0$, based on Proposition 2.3. The number of such steps is increased for $\frac{a}{D}<1$ to deal with the singularity in (5.4). In this way we compute approximating values $f_{i}$ for $f\left(\eta_{i}\right)$. [However the examples given below all are computed with equidistant $\eta_{i}=i h$, for $i=1,2, \cdots$ and $h>0$.]

The occurrence of $f\left(\eta_{i}\right)=0$ is tested by $\left|f_{i}\right|<\varepsilon_{1}$ for some control parameter $\varepsilon_{1}>0$ and correspondingly $f\left(\eta_{i}\right)>0$ and $f\left(\eta_{i}\right)<0$ have to be interpreted. The parameter $A$ is considered to be in $S^{+}$if for some $i>\bar{l}(\bar{l} \in \mathbb{N}$ is a given control parameter, sufficient large) $f\left(\eta_{i}\right)>0$ and $f\left(\eta_{k}\right)$ remains close to $f\left(\eta_{i}\right)$ for $\bar{l} \leq k \leq i$. To avoid misinterpretation, in particular for $\frac{a}{D} \gg 1$, when the self-similar solution is very flat in the vicinity of $\eta=0$ (see Figure 4), we also require $\eta_{i} \geq \eta_{s}$ where $\eta_{s}$ is the position of the shock in the limit case $D=0$ (see (3.10) and (4.5)).

To facilitate the detection of $A \in S^{-}, \beta$ is extended monotonically for $f<0$. The parameter $A$ is considered to be in $S^{-}$, if for some $i$, say $i=\bar{l}, f\left(\eta_{\bar{l}}\right)<0$ and $f\left(\eta_{i}\right)>0$ for all $i<\bar{l}$.

For $0 \leq \eta \leq \infty$, let $M(\eta)$ denote the mass for the given $\eta>0$, scaled by $1 / \omega_{N}$ (see also $(3.3))$ :

$$
M(\eta):=\int_{0}^{\eta} \beta(f(\eta)) \eta^{N-1} d \eta
$$

where

$$
M(\infty)=\frac{2 a}{N} .
$$

We approximate $M\left(\eta_{i}\right)$ by the trapezoidal rule as a measure of accuracy and use it in the case of singular reactions $(L<\infty)$ to detect convergence and estimate the position of the front $L$. 
This is done by the requirement $f\left(\eta_{i}\right)=0$ and mass error $=\frac{2 a}{N}-M\left(\eta_{i}\right)<\varepsilon_{2}$, where $\varepsilon_{2}>0$ is a control parameter. In the case $L=\infty$ we use the requirement $f\left(\eta_{i}\right)=0$ and $f^{\prime}\left(\eta_{i}\right)=0$. An alternative in the finite case would be the use of Proposition 2.5. The choice of the discretization parameter $h$ determines the accuracy of a solution in terms of $\varepsilon_{1}$ and $\varepsilon_{2}$.

The shooting algorithm could also be based on Problem (IVP). If we then interpret the numerical procedure in terms of the original variable $\eta$, this amounts to a grading of the mesh $\left\{\eta_{i}\right\}$. For $a / D<1$ this way may be preferable, but not for $a / D \gg 1$.

In Figures 2-4 we show the solution profiles for the following data:

$\beta(u):=u+\frac{\rho}{\Theta} k_{1}(u)_{+}^{p} ;$

$\rho=\Theta=0.5, k_{1}=3, p=0.5$;

$(u)_{+}^{p}$ is regularized by a straight line in $\left[0,10^{-14}\right]$;

$Q=2.5$.

Figure $2: D=3 \Rightarrow \lambda=0.2653$;

Figure 3: $D=0.15 \Rightarrow \lambda=5.3052$;

Figure 4: $D=0.009375 \Rightarrow \lambda=84.883$;

$h=10^{-4}, \varepsilon_{1}=10^{-8}, \varepsilon_{2}=10^{-4}$.

Figure 2. Self-similar solution for $\frac{a}{D}<1$. 
Figure 3. Self-similar solution for $\frac{a}{D}>1$.

Figure 4. Self-similar solution for $\frac{a}{D} \gg 1$. 
Acknowledgement. The authors wish to acknowledge L.A. Peletier (Leiden University) for a number of fruitful discussions and P.A.C. Raats (Institute for Soil-Fertility, Haren (Gr.), Netherlands) for bringing this problem to our attention. This work was completed while both authors enjoyed the support and hospitality of the Institute for Mathematics and its Applications.

\section{REFERENCES}

[AP1] Atkinson, F.V. \& L.A. Peletier, Similarity profiles of flows through porous media, Arch. Rational Mech. Anal. 42 (1971), pp. 369-379.

[AP2] Atkinson, F.V. \& L.A. Peletier, Similarity solutions of the nonlinear diffusion equation, Arch. Rational Mech. Anal. 54 (1974), pp. 373-392.

[B] Bear J., Dynamics of Fluids in Porous Media, American Elsevier, New York (1972) (also Dover Publ. Inc. 1988].

[VDK1] DuiJn, C.J. van \& P. Knabner, Solute transport in porous media with equilibrium and non-equilibrium multiple-site adsorption: Travelling waves, J. reine angewandte Math. 415, (1991), pp. 1-49.

[VDK2] DuiJn, C.J. van \& P. KNaBneR, Travelling waves in the transport of reactive solutes through porous media: Adsorption and binary in exchange - Part II, Transport in Porous Media 8 (1992), pp. $199-226$.

[FC] Freeze, R.A. \& J.A. Cherry, Groundwater, Prentice Hall, Englewood Cliffs (N.J.) (1979).

[W] Whiтнам, G.B., Linear and Nonlinear Waves, Wiley, New York (1974). 